\title{
Evaluation of Quality of Life and Oral Changes of Patients in Head and Neck Radiotherapy: Observational Study
}

\author{
Emilly Silva e Silva1, Gerlane Lima Oliveira1, Ana Carolina Carneiro Cardoso1, \\ Isabella Melo Brito Ferreira1, Marco Tulio Brazão-Silva ${ }^{2}$, Douglas Magno Guimarães ${ }^{1 *}$ (1) \\ ${ }^{1}$ Dental School, Pará University Center, Rua Nove de Janeiro, Belém, Pará, Brazil \\ ${ }^{2}$ Dental School, Montes Claros State University, Montes Claros, Minas Gerais, Brazil \\ Email: *douglas_guima@hotmail.com
}

How to cite this paper: e Silva, E.S., Oliveira, G.L., Cardoso, A.C.C., Ferreira, I.M.B., Brazão-Silva, M.T. and Guimarães, D.M. (2021) Evaluation of Quality of Life and Oral Changes of Patients in Head and Neck Radiotherapy: Observational Study. Journal of Cancer Therapy, 12, 641-653.

https://doi.org/10.4236/jct.2021.1211056

Received: October 20, 2021

Accepted: November 27, 2021

Published: November 30, 2021

Copyright $\odot 2021$ by author(s) and Scientific Research Publishing Inc. This work is licensed under the Creative Commons Attribution International License (CC BY 4.0).

http://creativecommons.org/licenses/by/4.0/

\begin{abstract}
Radiotherapy (RT) in the head and neck, despite coming from technological evolution, is challenged by the acute and late side effects of local irradiation, including permanent loss of saliva, osteoradionecrosis, dental caries induced by radiation and necrosis of the oral cavity, mucositis, xerostomia and secondary infections like candidiasis. Since these manifestations are limiting and the integrity of the vital organs contemplates the patient's general health status, the oral health-related quality of life (OHRQoL) can be significantly affected by the treatment of head and neck cancer (HNC), since cancer can cause important changes in vital functions related to communication, food and social contact, causing disturbances in the patient's life. An observational and longitudinal study was carried out with 16 cancer patients submitted to RT in the head and neck, between the years 2019-2020 with the objectives of collecting clinical and epidemiological data on the main oral changes caused by $\mathrm{RT}$ in the head and neck and correlating them with the impact on OHRQoL. To analyze the OHRQoL, the University of Washington's Quality of Life Assessment questionnaire (UW-QOL) was applied once a week for 4 weeks and to observe oral manifestations, a dental clinic file standardized by the researchers was applied. The main oral manifestations clinically observed were hyposalivation, trismus and oral mucositis. The UW-QOL presented pain, chewing and taste as the main complaints reported by patients, however, appearance, salivation and chewing showed statistically significant differences over the weeks. The main manifestations observed were hyposalivation, trismus and oral mucositis, the physical limitations resulting from these manifestations impact the OHRQoL of cancer patients in terms of appearance, salivation and chewing.
\end{abstract}




\section{Keywords}

Adverse Reactions, Head and Neck Neoplasms, Radiotherapy

\section{Introduction}

Head and neck cancer (HNC) is the common name for malignant tumor arising from the upper aerodigestive tract (oral and nasal cavities, pharynx and larynx), paranasal sinuses, salivary glands and thyroid gland [1]. Most malignant head and neck tumors originate in the epithelial cells lining the mucosa of the upper aerodigestive tract and are called squamous cell carcinoma (SCC). Head and neck squamous cell carcinomas have an annual incidence of 600,000 cases worldwide, with mortality in $40 \%-50 \%$ of cases [2]. It is the commonest HNC in men and fifth among all cancers, with an estimated risk of 10.69 new cases for every 100,000 men and 3.71 for every 8,100,000 women, being the thirteenth most incident type of cancer among females [3]. In Brazil, 37.120 new cases of HNC are estimated by Brazil's National Institute of Cancer (INCA) for 2020, as such considered the fourth most frequent type in Brazil. The interaction between genetic, behavioral and dietary factors predisposes to the onset and advancement of the disease. Smoking is the main risk factor, causing $22 \%$ of deaths from the disease [4].

In general, the literature considers surgery and radiotherapy (RT) to be the most effective methods used for the treatment of head and neck tumors, and chemotherapy and immunotherapy are important as auxiliary therapies [5]. In this context, in the head and neck region there are significant sequelae caused by local irradiation and consequent destruction of healthy tumor cells in this region, in addition to also presenting cytotoxic effects in the body arising from the metabolization of anticancer drugs [6]. Manifestations that occur during treatment or within 2 to 3 weeks after the RT cycle are considered acute effects. Late effects tend to present from weeks to years later, representing late tissue changes that result from the evolution or consolidation of acute lesions, including permanent saliva loss, osteoradionecrosis, radiation-induced dental caries, and oral cavity necrosis. The most frequent acute effects are mucositis, xerostomia (dry mouth) and secondary infections, such as candidiasis [6] [7]. Therefore, the quality of life related to oral health (OHRQoL) may be significantly affected for treatment of HNC, given that the social-emotional interactions are directly linked to the structural and functional integrity of the head and neck organs [8]. Specific tools were created to assess the OHRQoL of certain disease, the most frequently used specific instruments to assess patients with head and neck cancer are the European Organization for Research and Treatment of Cancer (EORTC), Quality of Life Questionnaire (QLQ-C30 and HN-35 modules) and the University of Washington's Quality of Life Assessment questionnaire (UW-QOL), with the last two presenting the best reports [8] [9]. The UW-QOL is a concise tool, easy 
to complete and interpret, and it was recently validated for the Brazilian Portuguese version for patients with head and neck cancer [10]. Fundamental general and specific aspects such as the impact on social interaction and dysphagia, respectively, are measured through these tools and widely inserted in cancer-related research to reveal patients' experiences in relation to the treatment and the disease [9].

Therefore, the general objective of this work is to verify the oral health-related quality of life of patients undergoing radiotherapy in the head and neck region, identifying the main oral changes that are related to radiotherapy in the head and neck region, the factors that interfere with quality of life and relating the number of radiotherapy sessions with the presence of oral manifestations.

\section{Methods}

\subsection{Study Design}

Observational and cross-sectional study carried out with 16 cancer patients submitted to RT in the head and neck at the Ophir Loyola Hospital (OLH), at the city of Belem-Pará, between the years December of 2019 and December 2020 .

\subsection{Study Population and Sampling}

Sixteen cancer patients diagnosed with HNC and undergoing cancer treatment at the OLH, followed by the hospital's dental service, were invited to participate in the research, in a randomized manner.

\subsection{Inclusion and Exclusion Criteria}

\subsubsection{Inclusion Criteria}

Patients of both sexes, aged between 18 and 99 years diagnosed with head and neck cancer and undergoing radiotherapy in this region.

\subsubsection{Exclusion Criteria}

Patients without the possibility of telephone location and not attending the outpatient appointment, patients and under palliative care and/or under chemotherapy.

\subsection{Procedures}

The researchers approached the patients in the RT clinic and explained the objectives, risks and benefits of the research. After consent was obtained by signing the Informed Consent Form (ICF), the data were collected through the UW-QOL, which is composed of 12 multiple-choice questions that address specific domains, such as: pain, appearance, activity, recreation, swallowing, chewing, speech, shoulders, palate, saliva, mood and anxiety. Each of the questions has three to five scored response options ranging from 0 to 100 points, with 0 being the worst condition (greater impact on health-related quality of life-HRQOL) and 100 indicating the best HRQOL [11] (Appendix). 
After application of the tool, the oral cavity was evaluated by two researchers together in order to clinically identify any abnormalities. All observations were recorded on a standardized form by the researchers consisting socio and behavioral information: age, gender, diagnosis of malignant neoplastic lesions, staging of lesions, Gy radiation doses, number of RT sessions performed, combination of treatments and the presence of habits such as tobacco and alcoholism. The researchers began following up patients with ongoing treatment. The assessment and completion of the questionnaire were applied to patients in the outpatient clinic before each RT session, with an average duration of 5 minutes and once a week, for 4 weeks, totaling 24 sessions.

\subsection{Data Analysis}

The data were grouped by weeks under RT session, sex, age and gender. The groups were compared for demographic and baseline characteristics using an X2 test for categorical variables (e.g., gender) and Student's t-test was used to compare scores between weeks. The association between these data and the possible quality of life-modifying factors was studied through bivariate analysis in GraphPad 5.0 for Mac (GraphPad Software, La Jolla, California, USA) for continuous variables. For QOL analysis, the chi-square was used. A P value less than 0.05 was considered statistically significant.

\section{Results}

The demographic profile of this research sample consisted of 16 patients evaluated with a diagnosis of head and neck cancer undergoing radiotherapy treatment being 11 men and 5 women with a mean age of 60 years, irradiated with an average dose of $200 \mathrm{cGy}$ (Table 1).

The most prevalent complaint was pain, swallowing and dysgeusia. Furthermore, between the thirteenth and eighteenth sessions, the highest frequency of complaints was observed (Image 1).

The criteria with the greatest statistical differences were appearance, chewing and salivation. Regarding appearance, deterioration was observed throughout the sessions, with the lowest levels above 19 sessions (average $=64.29$ ), with a statistical difference between above 13 sessions (average $=64.29)$ and below 12

Table 1. Representation of gender, age and mean radiation dose variables. Belém-Pará, $2019 / 2020$

\begin{tabular}{ccccc}
\hline & \multicolumn{4}{c}{ Radiotherapy sessions } \\
\cline { 2 - 5 } Variables & $\begin{array}{c}1-6 \\
\text { sessions }\end{array}$ & $\begin{array}{c}7-12 \\
\text { sessions }\end{array}$ & $\begin{array}{c}13-18 \\
\text { sessions }\end{array}$ & $\begin{array}{c}\text { sessions more } \\
\text { Masculine }\end{array}$ \\
Feminine & 4 & 2 & 2 & 3 \\
Mean age & $58(18-91)$ & $69(55-91)$ & $67(56-91)$ & $61(40-91)$ \\
Radiation dose (cGy) & $200(90-700)$ & $200(20-700) 200(90-700) 220(200-700)$ \\
\hline
\end{tabular}



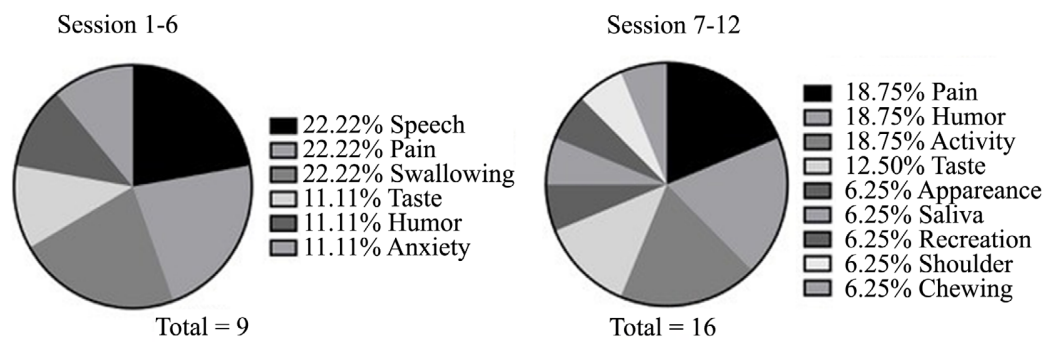

Session 13-18

Session 19 more
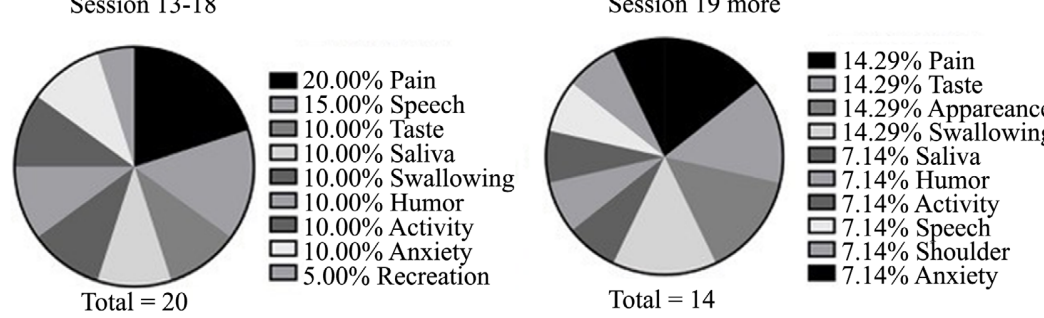

Image 1. Clinically observed oral manifestations during radiotherapy treatment due to the number of combinations.

sessions (average $=78.47$ ). The chewing factor showed a worsening with lower levels in the thirteenth and seventh sessions, with improvement from the nineteenth session (average $=78.57$ ) and statistically significant difference between $13-18$ (average $=28.57$ ) with $7-12$ weeks (average $=57.14$ ) and more than 19 sessions. In the salivation criterion, a reduction in values was observed throughout the sessions, with the worst values above 19 sessions (average $=38.14$ ), with a statistical difference for values from the twelfth radiotherapy session onwards (average $=28.57$ ).

In the pain criterion, a progressive worsening was observed throughout the sessions, with improvement after 19 sessions (average $=64.29$ ), but there was no difference between the periods. Regarding activity and recreation, respectively, low values were observed in all groups. And for the taste criterion, a reduction in values was observed throughout the sessions, with the worst values above 19 sessions (average $=47.71$ ). Therefore, it was observed that the criteria pain, activity, taste and recreation demonstrated a continuous reduction during the sessions.

Furthermore, for the swallowing criterion, low values were noted in all groups, with no statistical differences. For the speech and shoulder criteria, the scores were high for the groups, with no statistical difference. Mood and anxiety had high scores, however the first one kept a pattern and the second one showed a worsening after the sixth session (average $=57.14$ ), it improved after the twelfth session (average $=66.71$ ) and decreased again after the eighteenth session (average $=47.89$ ). The scores for each criterion are summarized in Table 2 and, for better visualization, they were grouped in column charts (Image 2).

The main clinical findings observed during treatment were hyposalivation, trismus and oral mucositis (Image 3). An improvement in hyposalivation and trismus was noted during the sessions, while OM became more frequent after the thirteenth session. In addition, candidiasis began to be observed from the seventh session onwards. 
Pain

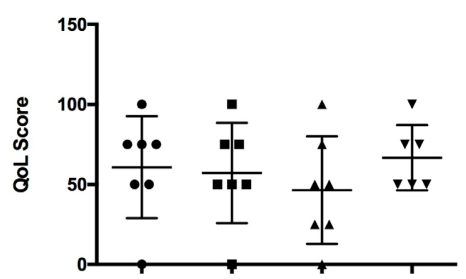

(a)

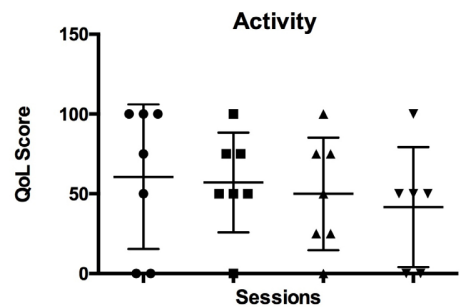

(c)

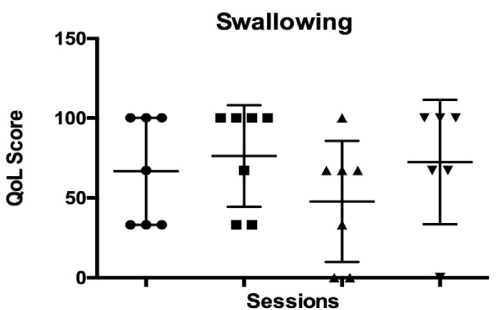

(e)

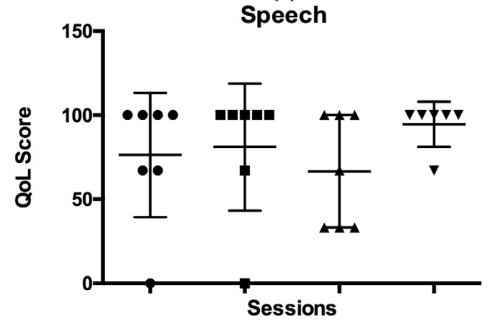

(g)

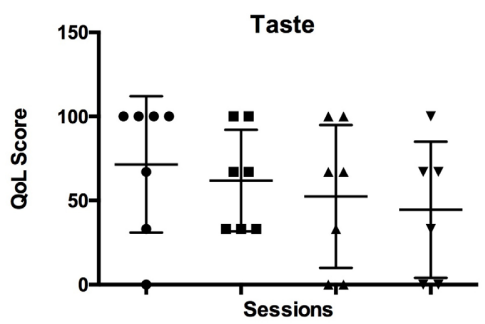

(i)

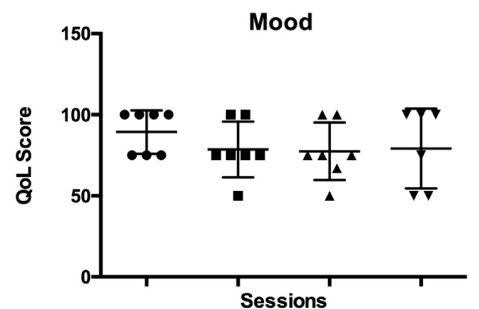

(k)
- Session 1-6

- Sessions 7-12

- Sessions 13-18

- Sessions 19-24

- Session 1-6

- Sessions 7-12

- Sessions 13-18

- Sessions 19-24

- Session 1-6

- Sessions 7-12

- Sessions 13-18

- Sessions 19-24

- Session 1-6

- Sessions 7-12

- Sessions 13-18

- Sessions 19-24

- Session 1-6

- Sessions 7-12

- Sessions 13-18

- Sessions 19-24

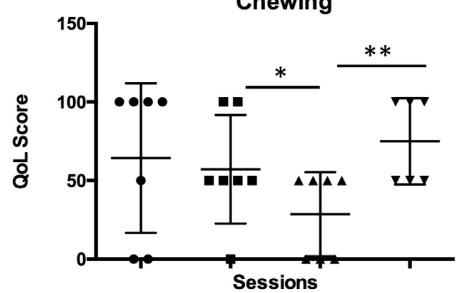

(f)

Shoulder

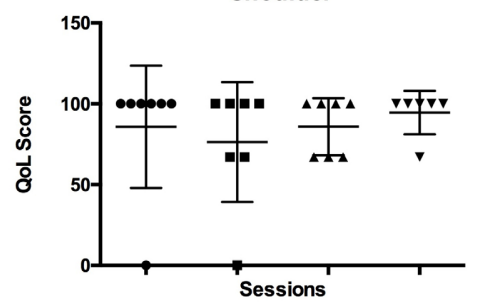

Session 1-6

- Sessions 7-12

- Sessions 13-18

- Sessions 19-24
- Session 1-6

- Sessions 7-12

- Sessions 13-18

v Sessions 19-24 (h)

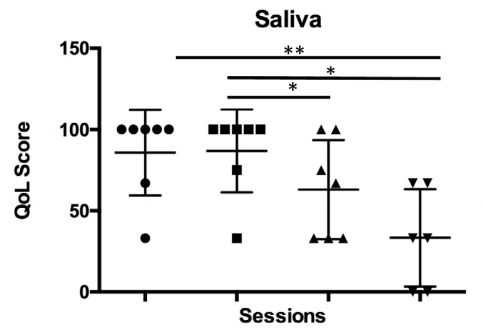

(j)

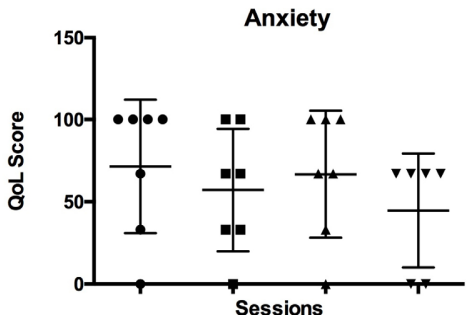

(1)

Image 2. Graphs of pains scores, saliva, recreation, appareance, chewing, activity, taste, mood, speech, shoulder, swallowing and anxiety of the UW-QOL as a function of radiotherapy sessions. ${ }^{*}, \mathrm{p}<0.05 ;{ }^{\star *},-<0.001$. 


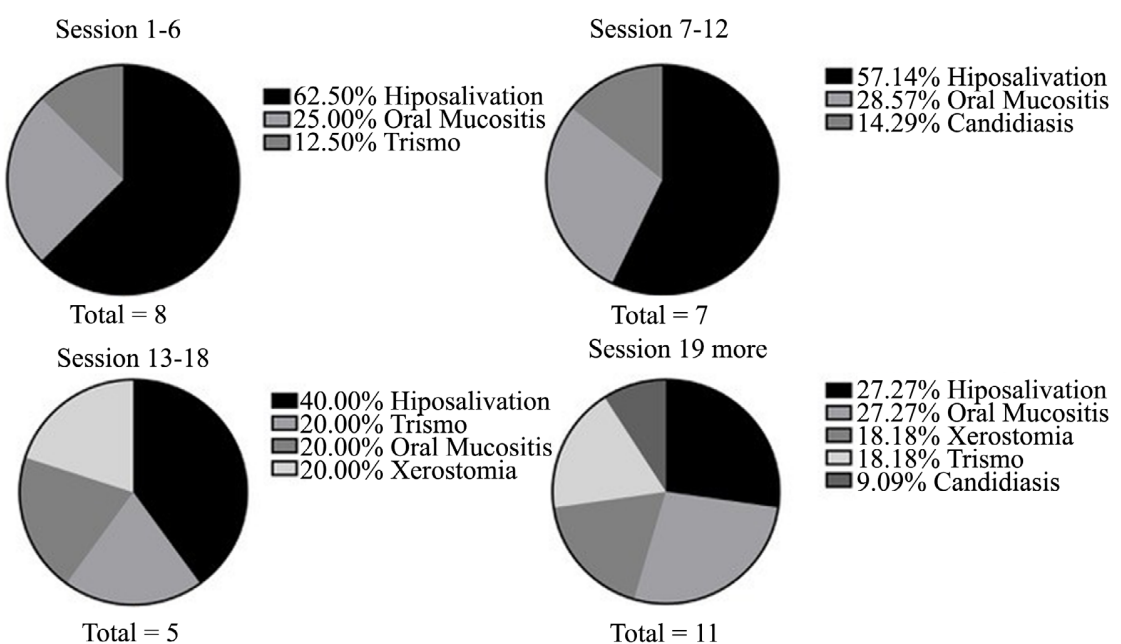

Image 3. Distribution of the main complaints reported by patients irradiated in the head and neck during the weeks. Belém-Pará, 2019/2020.

Table 2. UW-QOL of head and neck cancer patients evaluated during radiotherapy treatment. Belem-PA, 2019/2020.

\begin{tabular}{ccccc}
\hline \multirow{2}{*}{ Criteria } & \multicolumn{4}{c}{ Radiotherapy sessions } \\
\cline { 2 - 5 } & $1-6$ sessions & $7-12$ sessions & $13-18$ sessions & $>19$ sessions \\
\hline Pain & 60.71 & 57.14 & 46.43 & 64.29 \\
Appearance & 75 & 78.57 & 64.29 & 64.29 \\
Activity & 60.71 & 57.14 & 50 & 50 \\
Recreation & 53.57 & 64.29 & 50 & 50 \\
Swallowing & 66.57 & 76.14 & 47.71 & 76.29 \\
Chewing & 64.29 & 54.14 & 28.57 & 78.57 \\
Speech & 76.29 & 81 & 66.57 & 95.29 \\
Shoulder & 85.71 & 76.29 & 85.86 & 95.29 \\
Taste & 71.43 & 61.86 & 52.43 & 47.71 \\
Saliva & 85.71 & 86.86 & 63 & 38.14 \\
Mood & 89.29 & 78.57 & 77.43 & 78.57 \\
Anxiety & 71.43 & 57.14 & 66.71 & 47.86 \\
\hline
\end{tabular}

\section{Discussion}

In general, the integrity of vital organs contemplates the patient's general health, therefore, the oral side effects of RT and the limitations arising from these manifestations affect the health-related quality of life of these individuals. The demographic profile of this research sample showed prevalence in men, with a mean age of 60 years. Our results supported the hypothesis that side effects from RT manifested in the oral cavity affect the quality of life of patients with head and neck cancer, mainly due to the influence of appearance, chewing and salivation factors. The main clinically observed oral alterations were: hyposalivation, 
trismus and mucositis.

During head and neck radiotherapy, salivary glands are characterized as risky organs due to their radiosensitivity, which can lead to reduced salivary flow. Silva et al. [12] in their study, stated the existence of an inverse correlation between quality of life and salivary flow rate and, in their sample of 20 patients, $100 \%$ developed xerostomia after radiotherapy. Also, the literature explains that in addition to the physiologically unpleasant consequences of low salivary flow, such as pain, other undesirable adverse effects include impairment of chewing, swallowing and taste, severely compromising quality of life. In our research, carried out using the UW-QOL questionnaire, we also found the saliva variable with the greatest impact $(55.56 \%)$, showing a significant worsening throughout the treatment, and, corroborating the literature, pain, swallowing and taste they also remained as the main complaints observed throughout the treatment. Thus, our data confirm that the development of xerostomia negatively affects the quality of life of patients, as it generates pain and, concomitantly, difficulty in swallowing.

Hyposalivation is correlated with increased colonization by Candida sp., this relationship is explained by Suryawanshi et al. [13] because saliva functions as a mechanical maintenance barrier for the oral microbiota in healthy individuals with normal salivary gland function and a dry environment deficient in salivary enzymes and antibacterial proteins provides a favorable environment for fungal growth. Concomitantly with Suryawanshi et al., our findings interconnect the clinical manifestations of hyposalivation and candidiasis, as they demonstrated that hyposalivation is correlated with increased colonization by Candida sp and a consequent worsening in quality of life, as the presence of candidiasis from the seventh session onwards, concomitantly with the reduction of salivation values related to QOL.

Gwede et al. [14] carried out a study on changes in the quality of life in the first year of life of patients with head and neck cancer undergoing postoperative radiotherapy treatment. They noted that, during the six months of treatment, patients reported great difficulty in chewing and swallowing. Our research confirmed these data, since, in the UW-QOL questionnaire, a worsening was observed with lower levels in the thirteenth and seventh sessions in relation to the chewing factor, and it is possible to affirm that this was triggered by the presence of oral manifestations, such as trismus, mucositis and reduced salivary flow, which, in this period, presented high values, being $9.09 \%$ for trismus, $27.27 \%$ for mucositis and $54.55 \%$ for reduced salivary flow. Thus, the chewing criterion was compromised by the development of oral manifestations, such as MO, hyposalivation and trismus, resulting from the RT of HNC.

Aiming at health promotion, the role of the dental surgeon within the oncology team is to provide comprehensive care, preventing and acting on the deleterious oral conditions resulting from the therapy, being of great importance the knowledge about the main side effects arising from RT of head and neck. Almeida et al. [15], carried out a study with 30 patients in head and neck RT with 
the aim of evaluating the main sequelae of radiotherapy for six months, the results showed that the main oral manifestations observed were the development of trismus, with $75 \%$, followed by of mucositis, with $67 \%$, and reduced salivary flow, with $17 \%$. Accordingly, our research also presented trismus, hyposalivation and mucositis as the most frequent oral manifestations. In the first sessions, mucositis and hyposalivation had a prevalence of $20 \%$ and trismus of $60 \%$. However, during the sessions, other manifestations appeared and the percentages of hyposalivation and trismus gradually decreased, despite this, hyposalivation remained with the highest values in all sessions when compared to the other clinical findings. These results confirm that the most frequent side effects related to RT of HNC are hyposalivation, trismus and oral mucosites.

This study had significant limitations. The sample size reflects the low flow in the number of patients with HNC being treated at Ophir Loyola Hospital. In addition, the monitoring of patients had to be interrupted due to the current moment of the COVID-19 pandemic and the internal protocol for reducing the flow of employees and collaborators within the OLH without a scheduled return date, thus preventing the possibility of increasing the size research sample. However, the homogeneity of the present sample in terms of these variables and the treatment of all patients in the same hospital strengthens the validity of the comparisons made in this study.

It is suggested that further research be carried out to assess the main harmful effects of radiation on the head and neck region, as well as its impacts on oral health-related quality of life, with the intention that they corroborate with the results presented here, providing more subsidies to help health professionals to elaborate strategies to try to minimize the adverse effects of the treatment.

\section{Conclusion}

$\mathrm{RT}$ in the head and neck region presents significant sequelae caused by local irradiation, leading to a worsening in the patient's quality of life, especially regarding appearance, chewing and salivation. The most common complaints were pain, swallowing and taste, the main complaints reported by patients irradiated in the head and neck. This reinforces the need for specialized dental follow-up during radiotherapy treatment, in order to promote preventive measures that aim to minimize these sequelae, thus developing better coping with these patients during treatment and, consequently, better quality of life related to oral health.

\section{Conflicts of Interest}

The authors declare no conflicts of interest regarding the publication of this paper.

\section{References}

[1] Yeh, S. (2010) Radiotherapy for Head and Neck Cancer. Seminars in Plastic Surgery, 
24, 127-136. https://doi.org/10.1055/s-0030-1255330

[2] Leemans, C.R., Snijders, P.J.F. and Brakenhoff, R.H. (2018) The Molecular Landscape of Head and Neck Cancer. Nature Reviews Cancer, 18, 269-282. https://doi.org/10.1038/nrc.2018.11

[3] Ministry of Health and National Cancer Institute (2020) Ministério da Saúde, Instituto Nacional de Câncer. Estimativa 2020: incidência de câncer no Brasil. INCA, Rio de Janeiro.

[4] Forouzanfar, M.H., et al. (2016) Global, Regional, and National Comparative Risk Assessment of 79 Behavioural, Environmental and Occupational, and Metabolic Risks or Clusters of Risks, 1990-2015: A Systematic Analysis for the Global Burden of Disease Study 2015. The Lancet, 388, 1659-1724. https://doi.org/10.1016/S0140-6736(16)31679-8

[5] Freitas, D.A., et al. (2011) Oral Sequelae of Head and Neck Radiotherapy. Revista CEFAC, 13, 1103-1108. https://doi.org/10.1590/S1516-18462011005000071

[6] Ardito, F., et al. (2016) Expression of Salivary Biomarkers in Patients with Oral Mucositis: Evaluation by SELDI-TOF/MS. Oral Diseases, 22, 209-219. https://doi.org/10.1111/odi.12405

[7] Brook, I. (2020) Late Side Effects of Radiation Treatment for Head and Neck Cancer. Radiation Oncology Journal, 38, 84-92. https://doi.org/10.3857/roj.2020.00213

[8] Klein, J., Livergant, J. and Ringash, J. (2014) Health Related Quality of Life in Head and Neck Cancer Treated with Radiation Therapy with or without Chemotherapy: A Systematic Review. Oral Oncology, 50, 254-262. https://doi.org/10.1016/j.oraloncology.2014.01.015

[9] Iriya, P.M. DE O., Romaniszen, L.W., Fernandes, T.M.F. and Poleti, M.L. (2017) Health-Related Quality of Life of Patients with Squamous Cell Carcinoma: A Comparison According to Tumor Location. Brazilian Oral Research, 31, 1-7. https://doi.org/10.1590/1807-3107bor-2017.vol31.0105

[10] Vartanian J.G., et al. (2006) Brazilian-Portuguese Validation of the University of Washington Quality of Life Questionnaire for Patients with Head and Neck Cancer. Head \& Neck, 28, 1115-1121. https://doi.org/10.1002/hed.20464

[11] Ojo, B., et al. (2012) A Systematic Review of Head and Neck Cancer Quality of Life Assessment Instruments. Oral Oncology, 48, 923-937. https://doi.org/10.1016/j.oraloncology.2012.03.025

[12] Da Silva, R.G.B., et al. Evaluation of Xerostomia in Patients with Head and Neck Cancer Undergoing Radiotherapy Treatment. Revista Contexto \& Saúde, 17, 5-14.

[13] Suryawanshi, H., Ganvir, S.M., Hazarey, V.K. and Wanjare, V.S. (2012) Oropharyngeal Candidosis Relative Frequency in Radiotherapy Patient for Head and Neck Cancer. Journal of Oral and Maxillofacial Pathology, 16, 31-37. https://doi.org/10.4103/0973-029X.92970

[14] Gwede, C.K., Johnson, D., Sauder, B., Divan, H. and Trotti, A. (2001) Change in Quality of Life (QOL) and Function within the First Year in Patients Who Received Primary of Post-Operativeradiotheapy for Advanced Stage Head and Neck Cancer. International Journal of Radiation Oncology, 51, 408-409. https://doi.org/10.1016/S0360-3016(01)02576-7

[15] Almeida, F.C.S., et al. (2004) Dental Management of Oral Cancer Patients before and after Radiation Trerapy a Protocol Purpose. Pesquisa Brasileira em Odontopediatria e Clínica Integrada, 4, 25-31. 


\section{Appendix}

University of Washington Quality of Life Questionnaire (UW-QOL v3)

This questionnaire asks about your health and quality of life over the past seven days.

Please answer all of the questions by ticking one box for each question.

1) Pain. (Tick one box: [ ])

100 [ ] I have no pain.

75 [ ] There is mild pain not needing medication.

50 [ ] I have moderate pain-requires regular medication (e.g. paracetamol).

25 [ ] I have severe pain controlled only by prescription medicine (e.g. morphine).

0 [ ] I have severe pain, not controlled by medication.

2) Appearance. (Tick one box: [ ])

100 [ ] There is no change in my appearance.

75 [ ] The change in my appearance is minor.

50 [ ] My appearance bothers me but I remain active.

25 [ ] I feel significantly disfigured and limit my activities due to my appearance.

0 [ ] I cannot be with people due to my appearance.

3) Activity. (Tick one box: [ ])

100 [ ] I am as active as I have ever been.

75 [ ] There are times when I can't keep up my old pace, but not often.

50 [ ] I am often tired and have slowed down my activities although I still get out.

25 [ ] I don't go out because I don't have the strength.

0 [ ] I am usually in bed or chair and don't leave home.

4) Recreation. (Tick one box: [ ])

100 [ ] There are no limitations to recreation at home or away from home.

75 [ ] There are a few things I can't do but I still get out and enjoy life.

50 [ ] There are many times when I wish I could get out more, but I'm not up to it.

25 [ ] There are severe limitations to what I can do, mostly I stay at home and watch TV.

0 [ ] I can't do anything enjoyable.

5) Swallowing. (Tick one box: [ ])

100 [ ] I can swallow as well as ever.

67 [ ] I cannot swallow certain solid foods.

33 [ ] I can only swallow liquid food.

0 [ ] I cannot swallow because it "goes down the wrong way" and chokes me.

6) Chewing. (Tick one box: [ ])

100 [ ] I can chew as well as ever.

50 [ ] I can eat soft solids but cannot chew some foods.

0 [ ] I cannot even chew soft solids. 
7) Speech. (Tick one box: [ ])

100 [ ] My speech is the same as always.

67 [ ] I have difficulty saying some words but I can be understood over the phone.

33 [ ] Only my family and friends can understand me.

0 [ ] I cannot be understood.

8) Shoulder. (Tick one box: [ ])

100 [ ] I have no problem with my shoulder.

67 [ ] My shoulder is stiff but it has not affected my activity or strength.

33 [ ] Dor ou fraqueza em meu ombro me fizeram mudar meu trabalho

0 [ ] Pain or weakness in my shoulder has caused me to change my work/hobbies.

9) Taste. (Tick one box: [ ])

100 [ ] I can taste food normally.

67 [ ] I can taste most foods normally.

33 [ ] I can taste some foods.

0 [ ] I cannot taste any foods.

10) Saliva (Tick one box: [ ])

100 [ ] My saliva is of normal consistency.

67 [ ] I have less saliva than normal, but it is enough.

33 [ ] I have too little saliva.

0 [ ] I have no saliva.

11) Mood. (Tick one box: [ ])

100 [ ] My mood is excellent and unaffected by my cancer.

75 [ ] My mood is generally good and only occasionally affected by my cancer.

50 [ ] I am neither in a good mood nor depressed about my cancer.

25 [ ] I am somewhat depressed about my cancer.

0 [ ] I am extremely depressed about my cancer.

12) Anxiety. (Tick one box: [ ])

100 [ ] I am not anxious about my cancer.

67 [ ] I am a little anxious about my cancer.

33 [ ] I am anxious about my cancer.

0 [ ] I am very anxious about my cancer.

Which issues have been the most important to you during the past 7 days?

Tick [ ] up to 3 boxes.

[ ] Pain [ ] Swallowing [ ] Taste [ ] Appearance [ ] Chewing

[ ] Saliva [ ] Activity [ ] Sppech

[ ] Mood [ ] Recreation [ ] Shoulder [ ] Anxiety

General Questions

Compared to the month before you developed cancer, how would you rate your health-related quality of life? (Tick one box: [ ])

[ ] Much better

[ ] Somewhat better

[ ] About the same

[ ] Somewhat worse 
[ ] Much worse

In general, would you say your health-related quality of life during the past 7 days has been: (Tick one box: [ ]).

[ ] Outstanding

[ ] Very good

[ ] Good

[ ] Fair

[ ] Poor

[ ] Very poor

Overall quality of life includes not only physical and mental health, but also many other factors, such as family, friends, spirituality, or personal leisure activities that are important to your enjoyment of life. Considering everything in your life that contributes to your personal well-being, rate your overall quality of life during the past 7 days (Tick one box: [ ]).

[ ] Outstanding

[ ] Very good

[ ] Good

[ ] Fair

[] Poor

[ ] Very poor

Please describe any other issues (medical or nonmedical) that are important to your quality of life and have not been adequately addressed by our questions (you may attach additional sheets if needed). 